Chirurg 2019 90 (Suppl 2):S44 https://doi.org/10.1007/s00104-019-0861-0 Online publiziert: 13. Februar 2019

(c) Springer Medizin Verlag GmbH, ein Teil von Springer Nature 2019

\section{Originalpublikation}

Ikoma N, You YN, Bednarski BK et al (2017) Impact of recurrence and salvage surgery on survival after multidisciplinary treatment of rectal cancer. J Clin Oncol (35):2631-2638

Hintergrund. Die multimodale Therapie des Rektumkarzinoms trägt zu einer Reduktion der Lokalrezidivrate und zu einem verbesserten Langzeitüberleben bei. Der Stellenwert der chirurgischen Resektion beim Rezidiv nach onkologischer Resektion mit totaler mesorektaler Exzision (TME) ist bisher unzureichend untersucht.

Methoden. Insgesamt 735 Patienten mit einem lokal fortgeschrittenen Rektumkarzinom (cT3-cT4 oder $\mathrm{cN}+$ ), die am MD Anderson Cancer Center zwischen 1993 und 2008 eine präoperative Radiochemotherapie (RCT) und eine TME erhielten, wurden hinsichtlich Verteilung von Rezidiven, rezidivfreiem Überleben, Gesamtüberleben und Behandlung in der Rezidivsituation ausgewertet.

Ergebnisse. Der mediane Karzinomabstand des initialen Tumors von der Anokutanlinie betrug $5,0 \mathrm{~cm}$. Eine adjuvante Chemotherapie wurde in 624 Fällen $(84,9 \%)$ durchgeführt. Während einer medianen Nachbeobachtungszeit von 96 Monaten erlitten 151 Patienten (20,8 \%) ein Rezidiv (129 Patienten mit singulärem Rezidiv und 22 mit multiplen

Dieser Beitrag wurde erstpubliziert in Der Chirurg (2017) 88:974-974. https://doi.org/10. 1007/s00104-017-0524-y

\title{
Bedeutung der chirurgischen Therapie beim Rezidiv des Rektumkarzinoms
}

Rezidiven). Singuläre Rezidive fanden sich am häufigsten in der Lunge $(n=$ $55)$, gefolgt von hepatischen $(n=31)$ und lokalen Rezidiven ( $n=27)$. Rezidive traten im Median nach 17,5 Monaten, mit signifikanten Unterschieden ( $p=$ $0,001)$ zwischen hepatischen (11,2 Monate), pulmonalen (18,2 Monate) und lokalen Rezidiven (24,7 Monate), auf. $75,5 \%$ der Rezidive traten innerhalb der ersten 3 Jahre auf. Von 113 Patienten mit hepatischem, pulmonalem oder lokalem Rezidiv erhielten $57 \%$ eine erneute Resektion. Die Resektion führte $\mathrm{zu}$ einem signifikant verbesserten Langzeitüberleben bei Patienten mit pulmonalen (korrigiertes Hazard Ratio [HR] 0,25; $95 \%$-Konfidenzintervall [KI] 0,12-0,51; $p<0,001)$ oder hepatischen Rezidiven (HR 0,17; 95 \%-KI 0,05-0,62; $p=0,008)$, nicht jedoch bei Patienten mit Lokalrezidiv $(p=0,353)$.

Diskussion und Fazit. In dieser Arbeit traten Rezidive nach multimodaler Therapie beim fortgeschrittenen Rektumkarzinom am häufigsten in der Lunge auf. Die Resektion von Lokalrezidiven hatte im Gegensatz zur Resektion von Rezidiven in der Lunge und Leber keinen Einfluss auf das Gesamtüberleben. In einer kürzlich publizierten Arbeit von Harris et al. konnte bei 533 Patienten mit Lokalrezidiv durch eine Resektion in $59 \%$ der Fälle eine R0-Situation und für diese Fälle ein tumorspezifisches 5-JahresÜberleben von $44 \%$ erreicht werden [1]. Allerdings war die initiale Resektion und perioperative Therapie in der Arbeit von Harris et al. nicht standardisiert und es fehlen Angaben zur Lokalrezidivrate der initialen Rektumresektion.
Lokalrezidive nach perioperativer RCT und korrekt durchgeführter TME erfordern häufig eine technisch schwierige laterale Beckenwandresektion und sind möglicherweise Ausdruck eines biologisch aggressiveren Tumorverhaltens als Lokalrezidive nach unvollständiger TME. Die Lokalrezidivrate von $5 \%$ in den Stadien cT3-cT4 oder N+ lässt eine hohe chirurgische Qualität der TME annehmen und erklärt damit möglichweise den fehlenden Benefit der Resektion des Lokalrezidivs in der vorliegenden Arbeit. Dennoch sollten die retrospektiven Ergebnisse der vorliegenden Arbeit mit Vorsicht interpretiert werden und bei der Therapieplanung auch berücksichtigt werden, dass durch eine kurative Resektion des Lokalrezidivs die Lebensqualität des Patienten erhalten bleibt [2].

\section{Korrespondenzadresse}

\section{Prof. Dr. M. Anthuber}

Klinik für Allgemein-, Viszeral-, Transplantationschirurgie, Klinikum Augsburg Stenglinstr. 2, 86156 Augsburg, Deutschland matthias.anthuber@klinikum-augsburg.de

Interessenkonflikt. M. Schrempf und M. Anthuber geben an, dass kein Interessenkonflikt besteht.

\section{Literatur}

1. Harris CA, Solomon MJ, Heriot AG et al (2016) The outcomes and patterns of treatment failure after surgery for locally recurrent rectal cancer. Ann Surg 264:323-329

2. You YN, Habiba H, Chang GJ et al (2011) Prognostic value of quality of life and pain in patients with locally recurrent rectal cancer. Ann Surg Oncol 18:989-996 